\title{
A NON-LOCAL APPROACH FOR SAR AND INTERFEROMETRIC SAR DENOISING
}

\section{Charles-Alban Deledalle, Florence Tupin}

\author{
Institut Telecom, Telecom ParisTech \\ CNRS LTCI \\ Paris, France
}

\author{
Loïc Denis
}

\author{
Observatoire de Lyon, CNRS CRAL \\ UCBL, ENS de Lyon, Université de Lyon \\ Lyon, France
}

\begin{abstract}
Recently, non-local approaches have proved very powerful for image denoising. Unlike local filters, the nonlocal (NL) means introduced in [1] decrease the noise while preserving well the resolution. In the proposed paper, we suggest the use of a non-local approach to estimate single-look SAR reflectivity images or to construct SAR interferograms. SAR interferogram construction refers to the joint estimation of the reflectivity, phase difference and coherence image from a pair of two co-registered single-look complex SAR images. The weighted maximum likelihood is introduced as a generalization of the weighted average performed in the NL means. We propose to set the weights according to the probability of similarity which provides an extension of the Euclidean distance used in the NL means. Experiments and results are presented to show the efficiency of the proposed approach.
\end{abstract}

Index Terms - Non local means, maximum likelihood, interferometric synthetic aperture radar (InSAR)

\section{INTRODUCTION}

Non-local (NL) approaches have been proposed by Buades et al. in [1] to denoise images damaged by additive white Gaussian noise. While local filters lead to biases and resolution loss, NL techniques are known to efficiently reduce noise and preserve structures. Instead of combining neighboring pixels, the NL means average similar pixels. NL means assume there are enough redundant pixels (pixels having identical noisefree value) in the image to reduce the noise significantly. Let $v_{s}$ be the observed noisy value at site $s$ and $u_{s}$ its underlying noise-free value. NL means provide the estimate $\hat{u}_{s}$ defined by:

$$
\hat{u}_{s}=\frac{\sum_{t} w(s, t) v_{t}}{\sum_{t} w(s, t)}
$$

where $t$ is a pixel index and $w(s, t)$ is a data-driven weight depending on the similarity between pixels with

Thanks to the CNES, DGA and ONERA for providing the data. index $s$ and $t$. For robustness reasons, pixel similarity is evaluated by comparing surrounding patches around $s$ and $t$ with the use of the Euclidean distance:

$$
w(s, t)=\exp \left(-\frac{\sum_{k}\left(v_{s, k}-v_{t, k}\right)^{2}}{h}\right)
$$

where $s, k$ and $t, k$ denote respectively the $k$-th pixels in the patches centered on $s$ and $t$, and $h$ is a filtering parameter. Equation (1) and Equation (2) are well adapted to estimate noise-free values and to evaluate patch-similarity when the observed image is damaged by additive white Gaussian noise. We describe in the following how this approach can be extended to handle speckle noise.

\section{WEIGHTED MAXIMUM LIKELIHOOD}

The weighted average performed by NL means can be seen as a particular case of the weighted maximum likelihood (WML) estimation. ML based filters assume there exist redundant pixels and search the value which maximizes the likelihood over the set of redundant pixel values. Since this set is unknown, we propose to approach its indicator function with weights, which leads to the WML estimation:

$$
\hat{u}_{s}=\arg \max _{u} \sum_{t} w(s, t) \log p\left(v_{t} \mid u\right)
$$

If we consider SAR amplitude images damaged by multiplicative speckle noise, described by a Rayleigh distribution, the WML estimate is given by:

$$
\hat{R}_{s}=\frac{\sum_{t} w(s, t) A_{t}^{2}}{\sum_{t} w(s, t)}
$$

where $A$ is the noisy amplitude image and $R$ the noisefree reflectivity image. For InSAR data damaged by speckle noise, described by a zero-mean complex circular Gaussian distribution [2], the WML estimate is given 


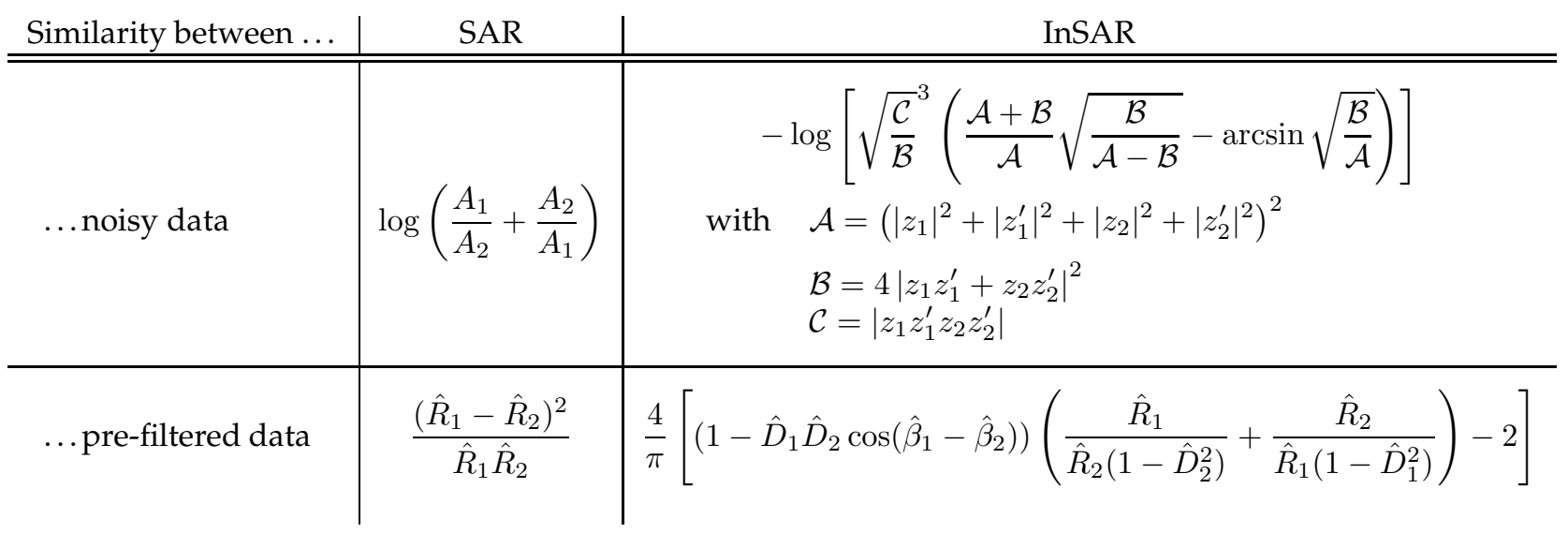

Table 1. Closed form expressions of the similarity criteria used to compare noisy patches and pre-filtered patches in the case of SAR and InSAR data.

by [3]:

$$
\begin{aligned}
\hat{R}_{s} & =\frac{a}{N}, \\
\hat{\beta}_{s} & =-\arg x, \\
\hat{D}_{s} & =\frac{|x|}{a} \\
\text { with } \quad a & =\sum_{t} w(s, t) \frac{\left|z_{t}\right|^{2}+\left|z_{t}^{\prime}\right|^{2}}{2}, \\
x & =\sum_{t}^{t} w(s, t) z_{t} z_{t}^{\prime *}, \\
N & =\sum_{t} w(s, t) .
\end{aligned}
$$

where $z$ and $z^{\prime}$ are two co-registered single-look complex SAR images. The parameters $R, D$ and $\beta$ are respectively the noise-free reflectivity, the coherence and the phase difference such that:

$$
\mathbb{E}\left\{\left(\begin{array}{c}
z \\
z^{\prime}
\end{array}\right)\left(z^{*} z^{\prime *}\right)\right\}=R\left(\begin{array}{cc}
1 & D e^{j \beta} \\
D e^{-j \beta} & 1
\end{array}\right) .
$$

\section{SETTING OF THE WEIGHTS}

As mentioned in Section 2, the weights should approach the indicator function of the set of redundant patches. In order to consider the statistical nature of the observed image, we use the probabilistic criterion introduced in [8], where the weights are set to :

$$
w(s, t)=\prod_{k}\left[p\left(v_{s, k}, v_{t, k} \mid u_{s, k}=u_{t, k}\right) p\left(u_{s, k}=u_{t, k}\right)\right]^{1 / h} .
$$

In the following the pixels $s_{s, k}$ and $t, k$ will be denoted respectively by ${ }_{1}$ and ${ }_{2}$. The first term $p\left(v_{1}, v_{2} \mid u_{1}=u_{2}\right)$ reflects the likelihood to have identical (unknown) noisefree values with respect to the observed noisy image.
A similar criterion has been applied in [9] to data damaged by additive white Gaussian noise. We extend here its definition as follows

$$
\begin{array}{r}
p\left(v_{1}, v_{2} \mid u_{1}=u_{2}\right)=\left|\frac{d \Phi}{d v_{1}}\left(v_{1}\right)\right|^{-1}\left|\frac{d \Phi}{d v_{2}}\left(v_{2}\right)\right|^{-1} \\
\int p\left(v_{1} \mid u_{1}=u\right) p\left(v_{2} \mid u_{2}=u\right) \mathrm{d} u
\end{array}
$$

where the Jacobian terms are introduced to take into account the change of variables due to a mapping function $\Phi$. The mapping $\Phi$ is introduced to obtain a dimensionless weight. The second term $p\left(u_{1}=u_{2}\right)$ tries to measure the prior probability to have equal noise-free values at sites ${ }_{1}$ and ${ }_{2}$. In [10], the authors propose to use the Kullback-Leibler divergence on an estimate $\hat{u}$ of $u$ as a statistical test of the hypothesis $u_{1}=u_{2}$. We have also observed good performances in practice of such a definition:

$$
p\left(u_{1}=u_{2}\right)=\exp \left[-\frac{1}{T} S D_{K L}\right]
$$

$$
\text { where } S D_{K L}=\int\left(p\left(v \mid \hat{u}_{1}\right)-p\left(v \mid \hat{u}_{2}\right)\right) \log \frac{p\left(v \mid \hat{u}_{1}\right)}{p\left(v \mid \hat{u}_{2}\right)} \mathrm{d} v
$$

where $\hat{u}$ is an estimate of $u$ and $T$ a filtering parameter. In practice, this estimate is refined iteratively by the use of the proposed algorithm. Both terms can be obtained in closed form in the case of SAR and InSAR data. The negation of their logarithm are given in Table 1. A more detailed description of the algorithm can be found in $[8,11]$.

Non-local approaches are known to leave a high variance in regions where there are too few redundant patches. In order to enforce a minimum amount of smoothing, different adaptive approaches have been proposed. In our filter, we suggest to select when required, the ten most similar pixels according to the similarity between the patches. 

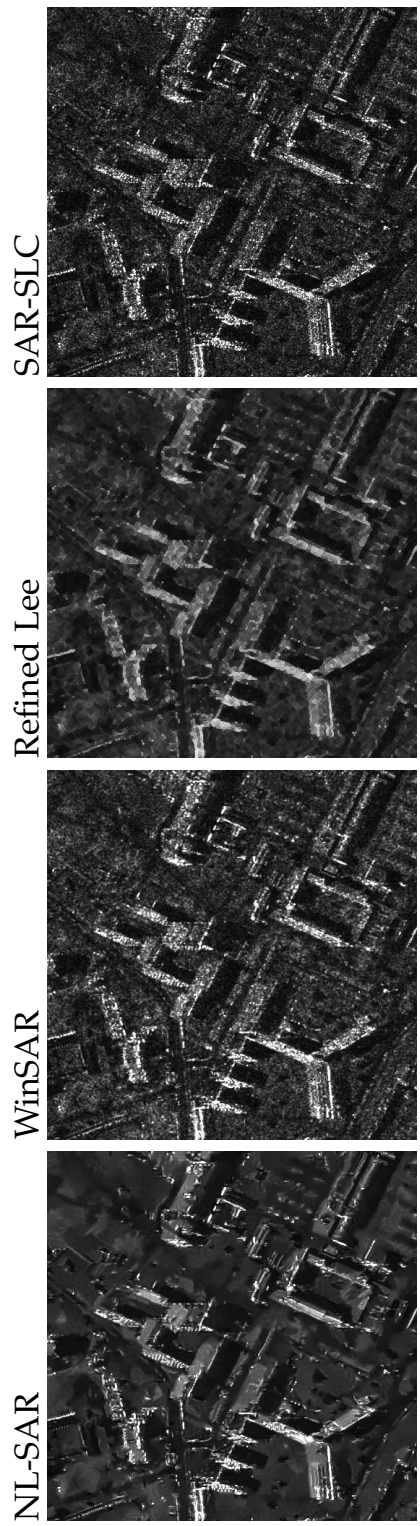

(a)
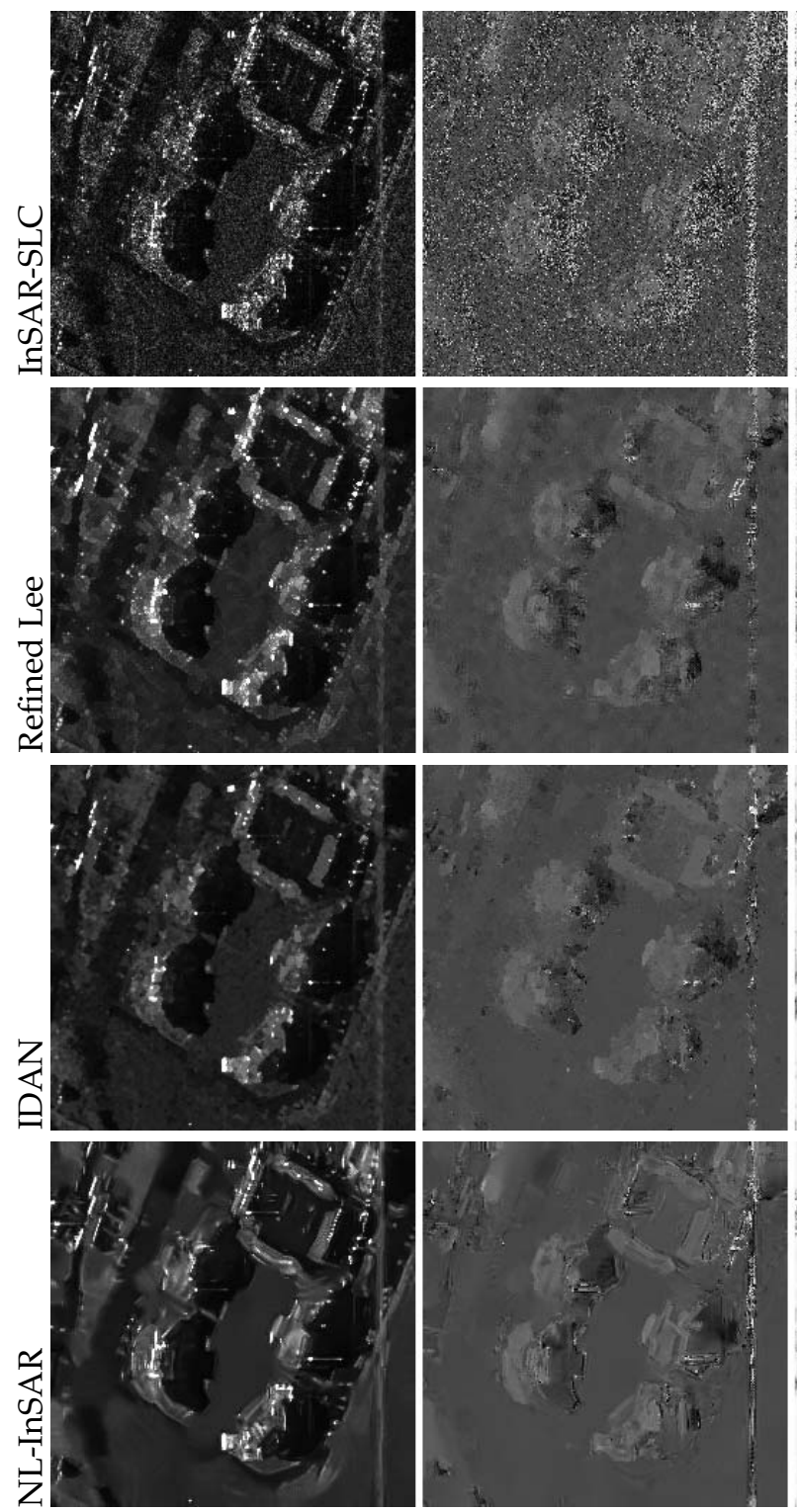

(b)

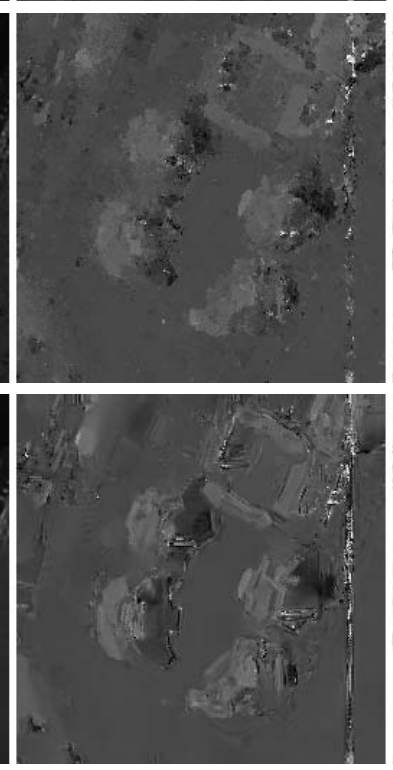

(c)

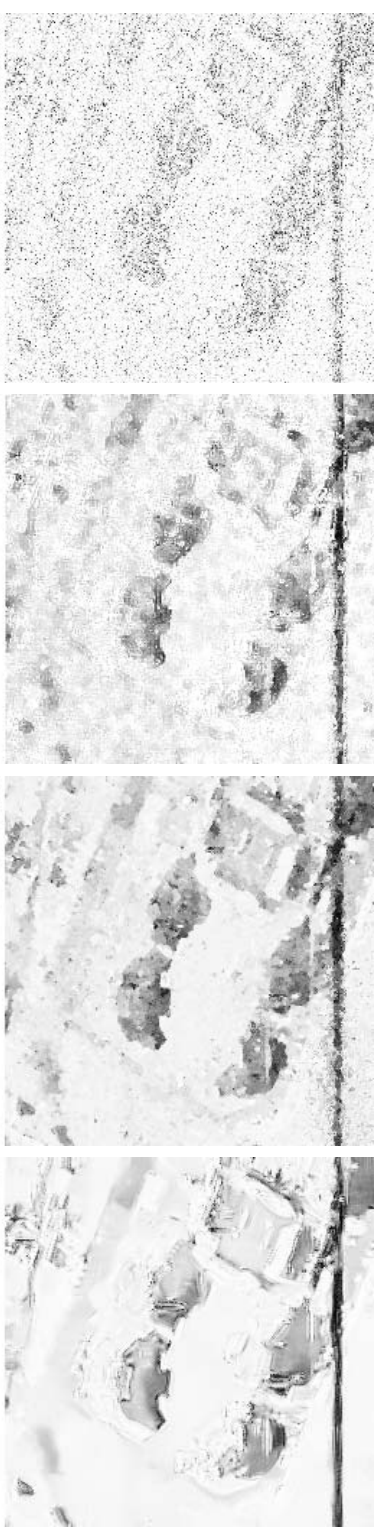

(d)

Fig. 1. (a) Reflectivity of Toulouse (France) sensed by TerraSAR-X (CDLR obtained by the single-look SAR image, the refined Lee filter [4], the WinSAR filter [5], and the NL-SAR filter. (b) Reflectivity, (c) phase difference and (d) coherence of Saint-Pol-sur-Mer (France) sensed by RAMSES (CDGA (C)ONERA, obtained from top to bottom by the SLC images (maximum likelihood estimator of [3]), the Refined Lee estimator [6], the IDAN estimator [7] and the NL-InSAR estimator.

\section{EXPERIMENTS AND RESULTS}

We have applied our methodology to SAR and InSAR data, resulting in two different filters denoted respectively by NL-SAR and NL-InSAR. Both use search windows of size $21 \times 21$, patches of size $7 \times 7$ and the parameters $h$ and $T$ are set as explained in [8]. The NL-SAR filter has been applied successfully on a single-look amplitude image of the CNES in Toulouse (France) sensed by TerraSAR-X. Results are given on Figure 1.a and compared to the original amplitude image, the Refined Lee filter [4] and the WinSAR filter [5]. The NL-InSAR filter has been applied on a pair of two co-registered single-look complex SAR images from Saint-Pol-surMer (France) sensed by RAMSES and provided by the CNES. The result is given on Figure 1.b,c,d and compared to the SLC images, the Refined Lee estimator [6] and the IDAN estimator [7]. The proposed NL-SAR 


\begin{tabular}{l|c|ccc} 
& SAR & \multicolumn{3}{|c}{ InSAR } \\
\hline \hline Channel & $R_{S A R}$ & $R_{\text {InSAR }}$ & $\beta$ & $D$ \\
\hline SLC & -4.42 & -2.75 & 3.36 & -1.19 \\
Refined Lee & 5.47 & 6.23 & 9.12 & 2.03 \\
WinSAR & 5.49 & - & - & - \\
IDAN & - & 5.00 & 7.88 & 0.33 \\
NL-(In)SAR & $\mathbf{7 . 4 6}$ & $\mathbf{9 . 0 2}$ & $\mathbf{1 3 . 0 4}$ & $\mathbf{6 . 9 2}$
\end{tabular}

Table 2. SNR values of estimated SAR and InSAR images using different estimators

and NL-InSAR filters provide the best quality images. The noise is well reduced while the resolution is well preserved.

To quantify the estimation qualities, Table 2 presents numerical results obtained on a resolution test pattern. The performance criterion used is the signal to noise ratio (SNR). Note that for the interferometric phase, we measure the SNR of the complex phase image $e^{j \hat{\beta}}$ to deal with phase wrapping. In case of SAR data, the results in terms of SNR are compared again with the Refined Lee, WinSAR and our proposed filter. In case of InSAR data, the results in terms of SNR are compared with the Refined Lee, IDAN and our proposed estimator. NL-SAR and NL-InSAR outperforms all the other filters for all components.

\section{CONCLUSION}

A new methodology is described which can be used to denoise SAR images and to construct SAR interferograms without significant loss of resolution. The proposed filters are based on non-local approaches. They combine similar pixels according to the similarity between their surrounding patches. The patch based similarity is defined with respect to the noise distribution model and is adapted to the specific nature of SAR data or InSAR data. Once the weights are computed, a weighted maximum likelihood estimation is performed for each pixel of the image. The process is then repeated to refine the weights and the quality of the final estimate. Visual and numerical results illustrate the efficiency of the algorithm.

\section{REFERENCES}

[1] A. Buades, B. Coll, and J.M. Morel, "A Non-Local Algorithm for Image Denoising," Computer Vision and Pattern Recognition, 2005. CVPR 2005. IEEE Computer Society Conference on, vol. 2, 2005.

[2] NR Goodman, "Statistical analysis based on a certain multivariate complex Gaussian distribution (an introduction)," Annals of Mathematical Statistics, pp. 152-177, 1963.

[3] MS Seymour and IG Cumming, "Maximum likelihood estimation for SAR interferometry," in The 1994 International Geoscience and Remote Sensing Symposium., 1994, vol. 4, pp. 2272-2274.

[4] J.S. Lee, "Refined filtering of image noise using local statistics," Computer graphics and image processing, vol. 15, no. 4, pp. 380-389, 1981.

[5] A. Achim, P. Tsakalides, and A. Bezerianos, "SAR image denoising via Bayesian wavelet shrinkage based on heavy-tailed modeling," IEEE Transactions on Geoscience and Remote Sensing, vol. 41, no. 8, pp. 1773-1784, 2003.

[6] J.S. Lee, SR Cloude, KP Papathanassiou, MR Grunes, and IH Woodhouse, "Speckle filtering and coherence estimation of polarimetric SAR interferometry data for forest applications," IEEE Transactions on Geoscience and Remote Sensing, vol. 41, no. 10 Part 1, pp. 2254-2263, 2003.

[7] G. Vasile, E. Trouvé, J.S. Lee, and V. Buzuloiu, "Intensity-Driven Adaptive-Neighborhood Technique for Polarimetric and Interferometric SAR Parameters Estimation," IEEE Transactions on Geoscience and Remote Sensing, vol. 44, no. 6, pp. 1609 1621, 2006.

[8] C.A. Deledalle, L. Denis, and F. Tupin, "Iterative Weighted Maximum Likelihood Denoising with Probabilistic Patch-Based Weights," IEEE Transactions on Image Processing, vol. 18, no. 12, pp. 26612672, 2009.

[9] Y. Matsushita and S. Lin, "A Probabilistic Intensity Similarity Measure based on Noise Distributions," in IEEE Conference on Computer Vision and Pattern Recognition, 2007. CVPR'07, 2007, pp. 1-8.

[10] J. Polzehl and V. Spokoiny, "Propagationseparation approach for local likelihood estimation," Probability Theory and Related Fields, vol. 135, no. 3, pp. 335-362, 2006.

[11] C.A. Deledalle, L. Denis, and F. Tupin, "NLInSAR : Non-Local Interferogram Estimation," Rapport de recherche Télécom ParisTech, 2009D021, 2009. 